\title{
Crecimiento anual de cuatro especies arbóreas con yemas DESNUDAS NATIVAS DE LOS BOSQUES TEMPLADOS HÚMEDOS DE PATAgONIA
}

\author{
CLAUDIA M. SOSA ${ }^{1,2}$ y JAVIER G. PUNTIERI ${ }^{1,3}$
}

\begin{abstract}
Summary: Annual growth of four naked-bud tree species from the temperate rainforests of Patagonia. Plant species inhabiting regions with low seasonality in climate tend to exhibit some morpho-architectural features that occur less frequently in species from regions with seasonal climate. This study was aimed at evaluating the primary growth of nursery-grown trees of four woody species native to the temperate rainforests of Patagonia, also known as Valdivian rainforests, where the degree of seasonality is lower than in other Patagonian forests. The selected species, Aextoxicon punctatum, Luma apiculata, Myrceugenia exsucca and Sophora microphylla, have naked buds, like many tropical species. Fenology, growth and branching of the main axis of marked plants were evaluated during one calendar year. Primary growth was markedly seasonal in all four species, as previously recorded for coexisting tree species with scaly buds. However, notable intra- and inter-specific differences in the onset and completion of growth were detected; L. apiculata and M. exsucca (Myrtaceae) exhibited higher intra-specific variations than the other two species. Our results indicate that several factors would be involved in regulating primary growth in these species, something found in species with continuous growth.
\end{abstract}

Key words: Architecture, Aextoxicon punctatum, Luma apiculata, Myrceugenia exsucca, primary growth, Sophora microphylla, Valdivian rainforest.

Resumen: Las especies vegetales que habitan en regiones con bajo nivel de estacionalidad en el clima suelen presentar rasgos morfo-arquitecturales que ocurren con menor frecuencia en especies de regiones con clima estacional. El objetivo de este estudio fue evaluar el crecimiento primario en vivero de cuatro especies leñosas típicas de los bosques templados húmedos de Patagonia, conocidos como selva valdiviana, donde el grado de estacionalidad es menos marcado que en otros bosques patagónicos. Las especies estudiadas, Aextoxicon punctatum, Luma apiculata, Myrceugenia exsucca y Sophora microphylla, comparten con muchas especies tropicales la posesión de yemas desnudas. Se evaluaron, durante un año calendario, la fenología, el crecimiento y la ramificación del eje principal de plantas marcadas. El crecimiento primario en estas especies fue marcadamente estacional, como en se ha registrado en especies arbóreas con yemas escamosas con las que coexisten. Sin embargo, se detectaron notables diferencias intra- e inter-específicas en el inicio y la finalización del crecimiento; $L$. apiculata y $M$. exsucca (Myrtaceae) presentaron mayores variaciones que las restantes dos especies. Nuestros resultados indican la intervención de varios factores reguladores del crecimiento primario en las especies estudiadas, como ocurre en especies con crecimiento continuo.

Palabras clave: Arquitectura, Aextoxicon punctatum, crecimiento primario, Luma apiculata, Myrceugenia exsucca, selva valdiviana, Sophora microphylla.

${ }^{1}$ INIBIOMA, Universidad Nacional del Comahue-Consejo Nacional de Investigaciones Científicas y Técnicas, Quintral 1250, 8400 Bariloche, Argentina.

2 Universidad Nacional de Formosa, Av. Gutnisky 3200, 3600 Formosa, Argentina. claudiasosa@comahue-conicet.gob.ar

${ }^{3}$ Universidad Nacional de Río Negro, Sede Andina, Mitre 630, 8400 Bariloche, Argentina. jgpuntieri@gmail.com 


\section{INTRODUCCIÓN}

A partir de la difusión de los estudios sobre la arquitectura de las plantas, se ha confirmado que aquellas especies que habitan en regiones con bajo nivel de estacionalidad en las condiciones climáticas presentan una serie de rasgos morfoarquitecturales que ocurren con mucha menor frecuencia en especies de regiones con clima claramente estacional (Grosfeld \& Barthélémy, 2004; Barthélémy \& Caraglio, 2007). Al no encontrarse limitadas por condiciones ambientales restrictivas en ningún momento del año, las primeras exhiben con más frecuencia las siguientes características: crecimiento continuo (es decir crecimiento primario no interrumpido endógenamente), yemas desnudas (sin catáfilos) y ramificación continua (con ramas en todos o gran parte de los nudos de los ejes) e inmediata (o siléptica, simultánea con el crecimiento del eje que las porta) (Hallé et al., 1978). Por otro lado, diferentes factores pueden haber determinado que algunas de las especies que hoy en día son calificadas como nativas de una región con clima estacional pertenezcan a linajes originados en condiciones climáticas menos restrictivas. A la inversa, especies calificadas como nativas de una región con clima no estacional, podrían pertenecer a linajes originados en condiciones climáticas estacionales (Aizen \& Ezcurra, 1998). Surge entonces el siguiente interrogante: ¿en qué medida los rasgos morfoarquitecturales de una especie son acordes con las condiciones climáticas de la región donde habita esa especie? Cambios en las condiciones climáticas podrían determinar que algunos de los rasgos seleccionados bajo condiciones climáticas pasadas no resultaran óptimos bajo las nuevas condiciones. Las consecuencias podrían ser negativas pero no necesariamente letales para una especie en esta situación. Por ejemplo, poseer yemas desnudas podría aumentar las probabilidades de muerte de ápice durante la estación desfavorable.

Los bosques templados húmedos de Sudamérica, también conocidos como selva valdiviana, son el resultado de una extensa historia de aislamiento, grandes cambios climáticos, eventos geológicos e intervenciones humanas de diferente índole (Donoso, 1993; Latorre et al., 2007). Como consecuencia, estos bosques poseen importantes niveles de endemismo y gran valor de conservación
(Ezcurra \& Brion, 2005). La selva valdiviana cubre amplios gradientes latitudinales $\left(33-55^{\circ} \mathrm{S}\right)$ y altitudinales (490-750 m s.n.m.) a ambos lados de la cordillera de Los Andes, y ocupa una superficie mucho mayor en Chile que en Argentina. En estos bosques, los árboles perennifolios alcanzan gran altura y poseen copas densas, con lo cual la intensidad de luz solar que alcanza el sotobosque es baja. El nivel de humedad es alto y los troncos de los árboles están cargados de lianas, pequeños helechos y musgos (Muñoz Schick, 1980). En la selva valdiviana la temperatura media anual varía entre $9{ }^{\circ} \mathrm{C}$ y $12{ }^{\circ} \mathrm{C}$, la temperatura mínima media es $>0{ }^{\circ} \mathrm{C}$ en todos los meses del año, las precipitaciones anuales alcanzan entre 1800-5000 mm, y las precipitaciones mensuales son siempre $>50 \mathrm{~mm}$ (Muñoz Schick, 1980). Puede afirmarse que, si bien el clima en este bioma oscila estacionalmente, impone menos limitaciones para el crecimiento vegetal a lo largo del año que el clima en otros biomas de la Patagonia (Conti, 1998). Dada su historia geológica, sus condiciones de humedad y temperatura y las especies que incluye, la selva valdiviana es un sitio de gran interés para el estudio de las relaciones filogenéticas entre las especies tropicales y las especies típicas de zonas templadas (Cabrera \& Willink, 1980).

Entre las especies de la selva valdiviana se encuentran representantes perennifolios de linajes ampliamente diversificados en regiones tropicales de Sudamérica como ser las familias Fabaceae y Myrtaceae (Aizen \& Ezcurra, 1998). En concordancia con este posible vínculo evolutivo con bosques de regiones tropicales y subtropicales, se ha observado la existencia de yemas desnudas (carentes de catáfilos o pérula) en varias especies nativas de la selva valdiviana (e.g. Gerea, 2008; Magnin et al., 2012). Otros aspectos de su desarrollo, como ser la fenología y dinámica del crecimiento primario no han sido investigados. Podría plantearse entonces la posibilidad de que las especies de la selva valdiviana presenten también otros atributos frecuentes en regiones tropicalessubtropicales como ser crecimiento continuo y ramificación continua y/o inmediata. En especies con yemas escamosas, las marcas dejadas por los catáfilos sobre el tallo pueden persistir por muchos años y permiten inferir la periodicidad del crecimiento primario y de la ramificación (e.g. Barthélémy et al., 1999). Pero en plantas con 


\section{M. Sosa y J. G. Puntieri - Crecimiento anual en especies arbóreas de Patagonia}

yemas desnudas la evaluación del crecimiento y la ramificación demanda observaciones frecuentes y repetidas de los mismos ejemplares durante un año. A partir del estudio de la fenología y el crecimiento primario de especies de la selva valdiviana con yemas desnudas, se espera encontrar, en concordancia con la morfología de las yemas y los orígenes evolutivos de estas especies, bajos niveles de circunscripción temporal del alargamiento de los brotes.

\section{Materiales y Métodos}

\section{Especies en estudio}

Se seleccionaron cuatro especies típicas de la selva valdiviana: Aextoxicon punctatum Ruiz \& Pav. (olivillo o tique; Aextoxicaceae), Luma apiculata (DC.) Burret (arrayán o quetri; Myrtaceae), Myrceugenia exsucca (DC.) O. Berg. (patagua o pitra; Myrtaceae), y Sophora microphylla Aiton (pelú; Fabaceae). Estas especies se conocen en cuanto a sus atributos forestales (producción y calidad de madera) y ecológicos (Donoso, 2006). Todas ellas habitan en zonas húmedas (fondos de valle próximos a cuerpos de agua dulce o al mar) a menos de $900 \mathrm{~m}$ de altitud, y presentan yemas apicales desnudas (Fig. 1).

Se seleccionaron 20 ejemplares de cada especie desarrollados en el vivero forestal de la Asociación Civil Sembrar, ubicado a $12 \mathrm{~km}$ del centro de la ciudad de San Carlos de Bariloche (41 $07^{\circ} 15^{\prime \prime} \mathrm{S}, 71^{\circ} 15^{\prime} 06^{\prime \prime} \mathrm{O} ; 770$ m s.n.m.). Este sitio fue considerado apto para la realización de observaciones repetidas de los mismos ejemplares durante un año completo debido a su accesibilidad y a la abundancia de ejemplares en buen estado sanitario. Las plantas marcadas ocupaban una superficie de alrededor de $10 \mathrm{~m}^{2}$, ubicada debajo del dosel formado por árboles de Nothofagus antarctica (G. Forster) Oersted (ñire) con cobertura de alrededor del $50 \%$ (medida a partir de fotografías hemisféricas tomadas con una cámara con lente ojo de pez). Se definió esta localización a fin de evitar que las plantas sufrieran los efectos de heladas (considerando que el vivero se encuentra a mayor altitud que sus hábitats naturales). Al momento de iniciado este estudio cada planta se encontraba en una maceta de entre 2 y 5 litros conteniendo el suelo de origen volcánico típico de la Patagonia andina. Las macetas se encontraban distribuidas al azar en la superficie asignada y con riego controlado. Estas plantas fueron mantenidas en el mismo vivero desde el inicio de su desarrollo, y en las mismas macetas a fin de evitar los efectos del transplante sobre las raíces y el crecimiento aéreo. En noviembre de 2012, cuando se seleccionaron los ejemplares, éstos tenían seis años de edad y, en promedio, $98,6 \mathrm{~cm}$ de altura y $11,4 \mathrm{~mm}$ de diámetro basal del tronco (Tabla 1; Fig. 2A). Se registraron temperatura y humedad ambiental cada cuatro horas ( 1 h, 5 hs, 9 hs, 13 hs, 17 hs y 21 hs) mediante un sensor THD4520 I Data Logger Thermobutton instalado en el vivero. Se consideraron "diurnos" los valores de temperatura y humedad registradas a las 9 hs, 13 hs y 17 hs de cada día y "nocturnos" aquellos registrados a las $1 \mathrm{~h}, 5 \mathrm{hs}$ y $21 \mathrm{hs}$.

\section{Fenología y dinámica de crecimiento}

A principios de noviembre de 2013 se marcó el brote terminal del tronco o eje principal (aquel que alcanzaba mayor altura) de cada ejemplar utilizando cintas plásticas. En aquellos individuos que no presentaban un eje principal único y claramente definido, se eligió al azar un eje de entre los más altos, y sobre éste se marcó el brote terminal. El brote marcado de cada planta será designado, en lo sucesivo, brote portador (Fig. 2B). Entre noviembre de 2013 y abril de 2014 se registró quincenalmente la longitud del brote nuevo (referido en lo sucesivo simplemente como brote) desarrollado a partir de la yema más distal del brote portador de cada planta con un calibre digital $(0,1 \mathrm{~mm}$ de precisión). Entre abril y noviembre de 2014 se continuaron realizando observaciones periódicas de las plantas

Tabla 1. Media y error estándar (EE) de la altura y el diámetro basal iniciales de las plantas

de Aextoxicon punctatum, Luma apiculata,

Myrceugenia exsucca y Sophora microphylla al inicio de la medición en vivero.

\begin{tabular}{|lcccc|}
\hline \multirow{2}{*}{ Especie } & \multicolumn{2}{c}{ Altura $(\mathrm{cm})$} & \multicolumn{2}{c|}{ Diámetro $(\mathrm{mm})$} \\
& Media & $\mathrm{EE}$ & Media & EE \\
\hline Aextoxicon punctatum & 78,7 & 2,13 & 6,8 & 0,20 \\
Luma apiculata & 114,6 & 2,17 & 16,0 & 0,46 \\
Myrceugenia exsucca & 96,6 & 1,33 & 12,8 & 0,47 \\
Sophora microphylla & 104,3 & 2,97 & 10,0 & 0,37 \\
\hline
\end{tabular}



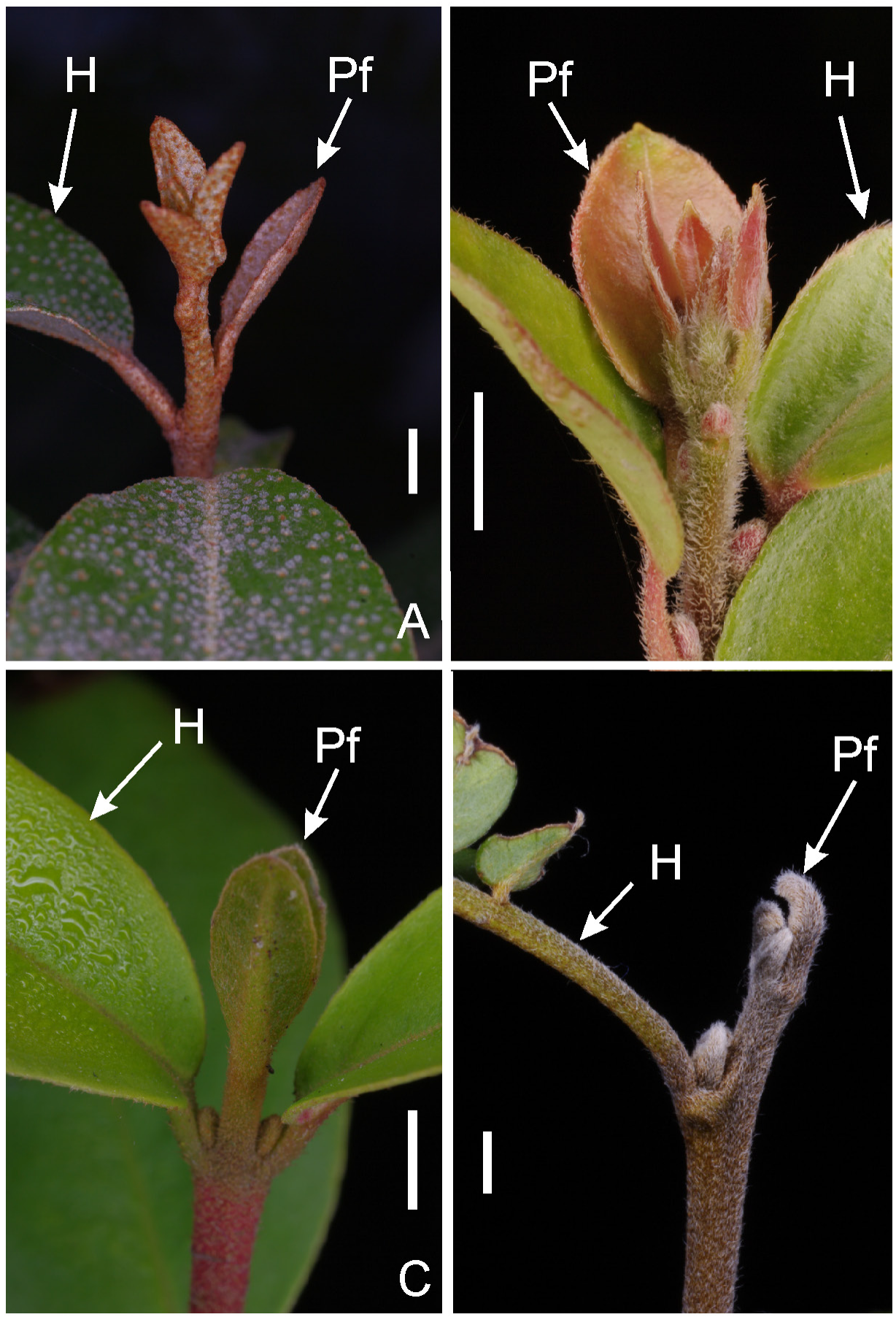

Fig. 1. Extremo distal de un brote de (A) Aextoxicon punctatum, (B) Luma apiculata, (C) Myrceugenia exsucca y (D) Sophora microphylla. En cada caso se indican: una hoja correspondiente al último nudo desarrollado antes de la formación de la yema apical $(\mathrm{H})$, y un primordio foliar (Pf) correspondiente al primer nudo de la yema apical. Todas las fotografías fueron tomadas entre los meses de junio y agosto. En el caso del brote (B), Luma apiculata, uno de los primordios del primer nudo de la yema apical fue cortado para facilitar la observación de primordios más internos. Escala: $1 \mathrm{~cm}$. 


\section{M. Sosa y J. G. Puntieri - Crecimiento anual en especies arbóreas de Patagonia}

a fin de detectar eventuales producciones de nuevos órganos. La longitud fue medida desde el extremo proximal del brote nuevo hasta la posición de su meristema apical; dicha posición debió ser estimada por visualización externa del ápice. El extremo proximal del brote nuevo fue marcado finamente con un marcador indeleble. En aquellos casos en los que el brote medido sufrió muerte apical durante su crecimiento con formación en el mismo período de un brote relevo, se continuó midiendo el brote relevo (Fig. 2C). Cuando no se formó tal relevo, el crecimiento del brote se consideró finalizado luego de la muerte del ápice.
Se calculó la tasa de alargamiento relativo (TAR) de cada brote para cada quincena de la estación de crecimiento, mediante la siguiente ecuación:

$\mathrm{TAR}=\log ($ longitud en la quincena $(\mathrm{n}+1))-\log$ (longitud en la quincena (n)).

Con el fin de definir objetivamente los tiempos inicial y final del alargamiento de los brotes, se obtuvo el porcentaje de cambio de longitud mínimo a partir del cual se consideró que se produjo el alargamiento de un brote entre dos mediciones sucesivas. Para ello se adoptó un error de medición que incluyó tanto el error del instrumento de medición (calibre) como el error de apreciación

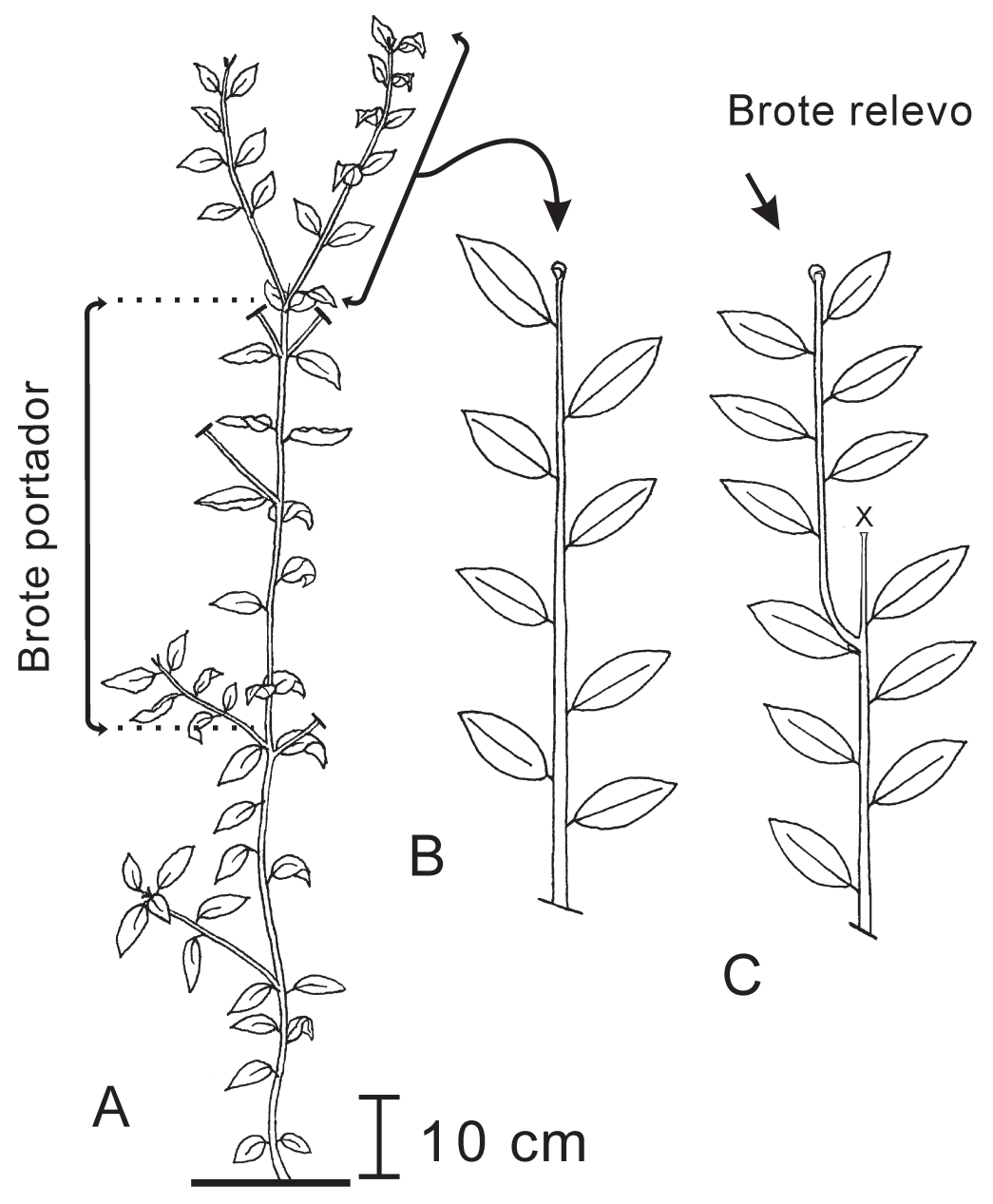

Fig. 2. Esquemas de: (A) un ejemplar modelo utilizado en el estudio en el cual se indica el brote portador, (B) un brote anual principal de ese ejemplar, y (C) un brote anual principal con muerte apical que ha desarrollado un relevo de origen axilar. Las líneas negras en el extremo de algunas ramas indican que parte de éstas no fueron ilustradas para facilitar la interpretación del dibujo. X: ápice muerto. 
del observador. Siguiendo el criterio adoptado en trabajos previos, en los que se realizaron mediciones repetidas de brotes de árboles a lo largo del tiempo con el mismo instrumento de medición, se consideró que todo cambio de longitud entre mediciones sucesivas menor del 6\% se debió al error asociado a la medición y no a un cambio de la longitud de un brote (Puntieri et al., 1998; Stecconi, 2006). Cada vez que se verificó esta última situación, se les asignó a las dos fechas de medición el promedio entre los dos valores medidos. Se determinó como quincena de inicio del alargamiento de un brote aquella en la cual se detectó un incremento en longitud mayor al error de medición mencionado, y como quincena de finalización del alargamiento a aquella a partir de la cual la variación en la longitud del brote no superó el error de medición en el transcurso de cuatro semanas. Se evaluó la duración del período de alargamiento de cada brote en quincenas, desde la de inicio hasta la de finalización del alargamiento.

Para las variables longitud del brote, número de entrenudos, diámetro y duración del crecimiento del brote anual, se evaluó si las distribuciones de frecuencias muestrales se ajustaban a una distribución teórica normal (para variables continuas, como la longitud, el diámetro y la duración del crecimiento) o binomial (para variables discretas, como el número de entrenudos) mediante el test de Kolmogorov-Smirnov; cuando se registraron desviaciones de las distribuciones teóricas, los datos fueron transformados en logaritmos naturales. La homogeneidad de varianzas se comprobó mediante el test de Levene.

\section{Resultados}

El crecimiento de todas las plantas estuvo limitado al período entre mediados de primavera e inicio de otoño. El crecimiento de varios brotes por especie se interrumpió por la muerte del ápice seguida de la formación, en las semanas siguientes, de un brote relevo derivado de una de las yemas axilares del brote en crecimiento. Este desarrollo de una rama de relevo se registró en cinco individuos de A. punctatum, 18 de L. apiculata, 16 de M. exsucca y dos de $S$. microphylla. No se desarrollaron otras ramas a partir de los brotes medidos en el período de observaciones. En ningún caso se observó el inicio de nuevos órganos entre la segunda mitad de abril y noviembre de 2014. Los promedios, así como los valores máximo y mínimo de las variables longitud, número de entrenudos y diámetro de los brotes de cada especie al finalizar su crecimiento primario están indicados en la Tabla 2.

Para A. punctatum, el inicio de alargamiento se produjo en la primera quincena de noviembre de 2013 en la mayoría de los individuos, mientras que unos pocos iniciaron el alargamiento a fines de noviembre (Fig. 3A). La finalización del alargamiento en esta especie se produjo entre la primera quincena de enero y la primera de abril de 2014 (Fig. 3A), y la duración del alargamiento de los brotes marcados abarcó entre 6 y 11 quincenas (Fig. 3B). La mayoría de los individuos de $A$. punctatum presentaron un pico en la TAR en la primera quincena de noviembre y un segundo pico en diciembre de 2013. Las variaciones posteriores fueron menos generales, aunque se destacó una

Tabla 2. Media, error estándar (EE) y valores máximo (Máx) y mínimo (Mín) registrados para las variables longitud, número de entrenudos y diámetro basal de los brotes anuales medidos en plantas de Aextoxicon punctatum, Luma apiculata, Myrceugenia exsucca y Sophora microphylla.

\begin{tabular}{|lcccccccccccccc|}
\hline \multicolumn{1}{|c}{ Especie } & \multicolumn{3}{c}{ Longitud $(\mathrm{mm})$} & \multicolumn{4}{c}{ Número de entrenudos } & \multicolumn{3}{c|}{ Diámetro basal $(\mathrm{mm})$} \\
& Media & EE & Máx & Mín & Media & EE & Máx & Mín & Media & EE & Máx. & Mín \\
\hline A. punctatum & 117,7 & 16,75 & 295,0 & 30,1 & 4,9 & 0,38 & 8 & 3 & 2,9 & 0,15 & 4,2 & 1,7 \\
L. apiculata & 50,3 & 8,70 & 140,8 & 14,4 & 5,9 & 0,63 & 12 & 3 & 1,5 & 0,09 & 2,4 & 1,1 \\
M. exsucca & 26,1 & 3,62 & 54,9 & 3,1 & 4,3 & 0,48 & 7 & 1 & 1,5 & 0,09 & 2,2 & 0,9 \\
S. microphylla & 31,1 & 7,81 & 104,7 & 2,7 & 3,5 & 0,53 & 8 & 1 & 2,1 & 0,10 & 2,9 & 1,6 \\
\hline
\end{tabular}




\section{Inicio y Finalización del crecimiento}

\section{Duración del alargamiento}

\section{Aextoxicon punctatum}
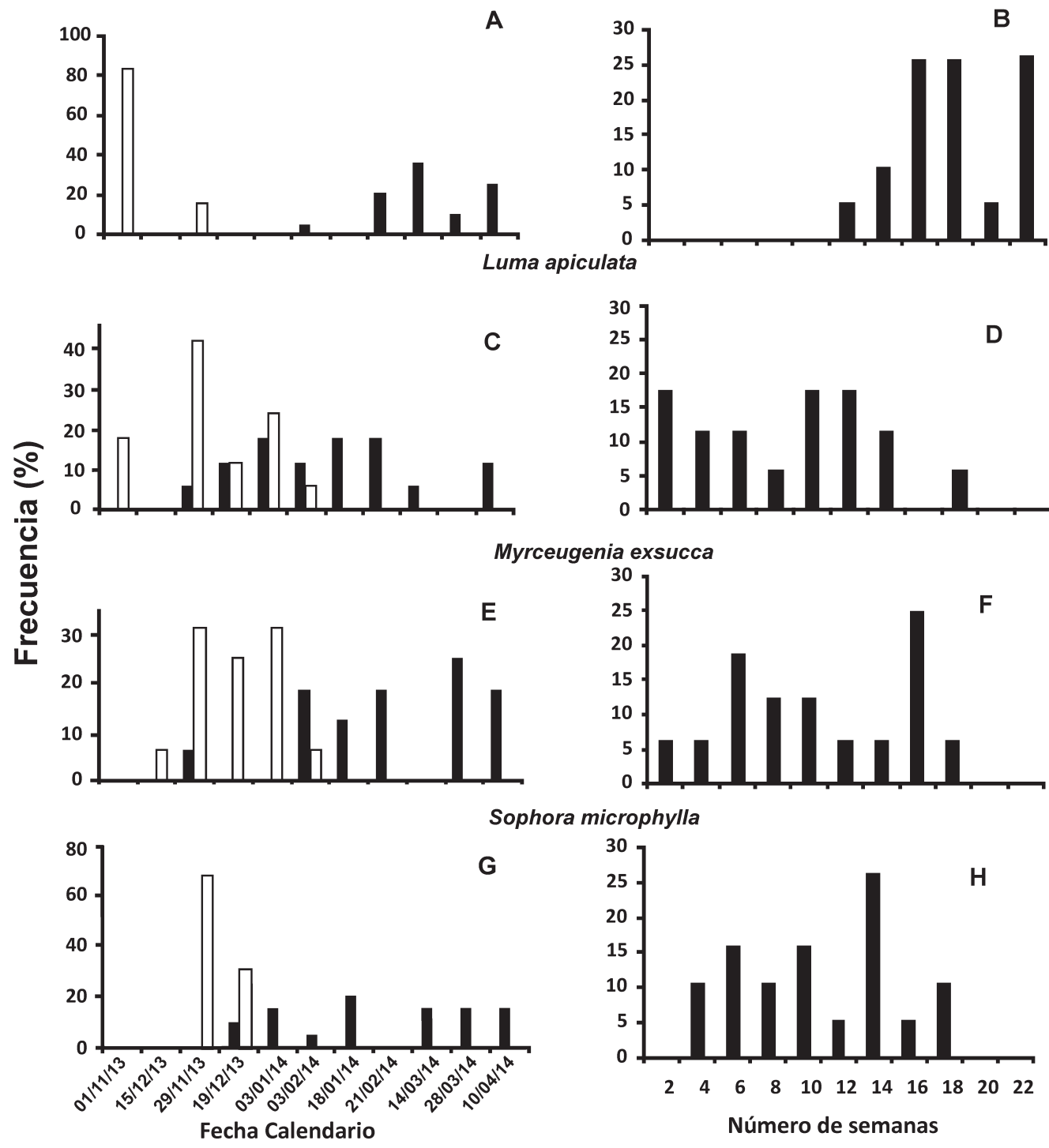

Fig. 3. A, C, E y G: distribuciones de frecuencias de las fechas de inicio (barras blancas) y de finalización (barras negras) del alargamiento de brotes anuales de Aextoxicon punctatum (A-B), Luma apiculata (C-D), Myrceugenia exsucca (E-F) y Sophora microphylla (G-H) en el período de crecimiento 2013-2014. B, D, F y $\mathrm{H}$ : distribuciones de frecuencias de la duración del alargamiento de los mismos brotes anuales.

notable disminución en la TAR de todas las plantas a principios de febrero de 2014 (Fig. 4).

El alargamiento de la mayoría de las plantas de L. apiculata marcadas se inició entre la primera quincena de noviembre de 2013 y la primera quincena de enero de 2014 (Fig. 3C). La finalización del alargamiento se produjo entre la segunda quincena de noviembre de 2013 y la primera de abril de 2014 (Fig. 3C), y la duración del alargamiento varió entre 1 y 9 quincenas (Fig. 3D). Para la mayoría de los individuos de L. apiculata, la TAR presentó su valor máximo en noviembre de 
Bol. Soc. Argent. Bot. 51 (4) 2016

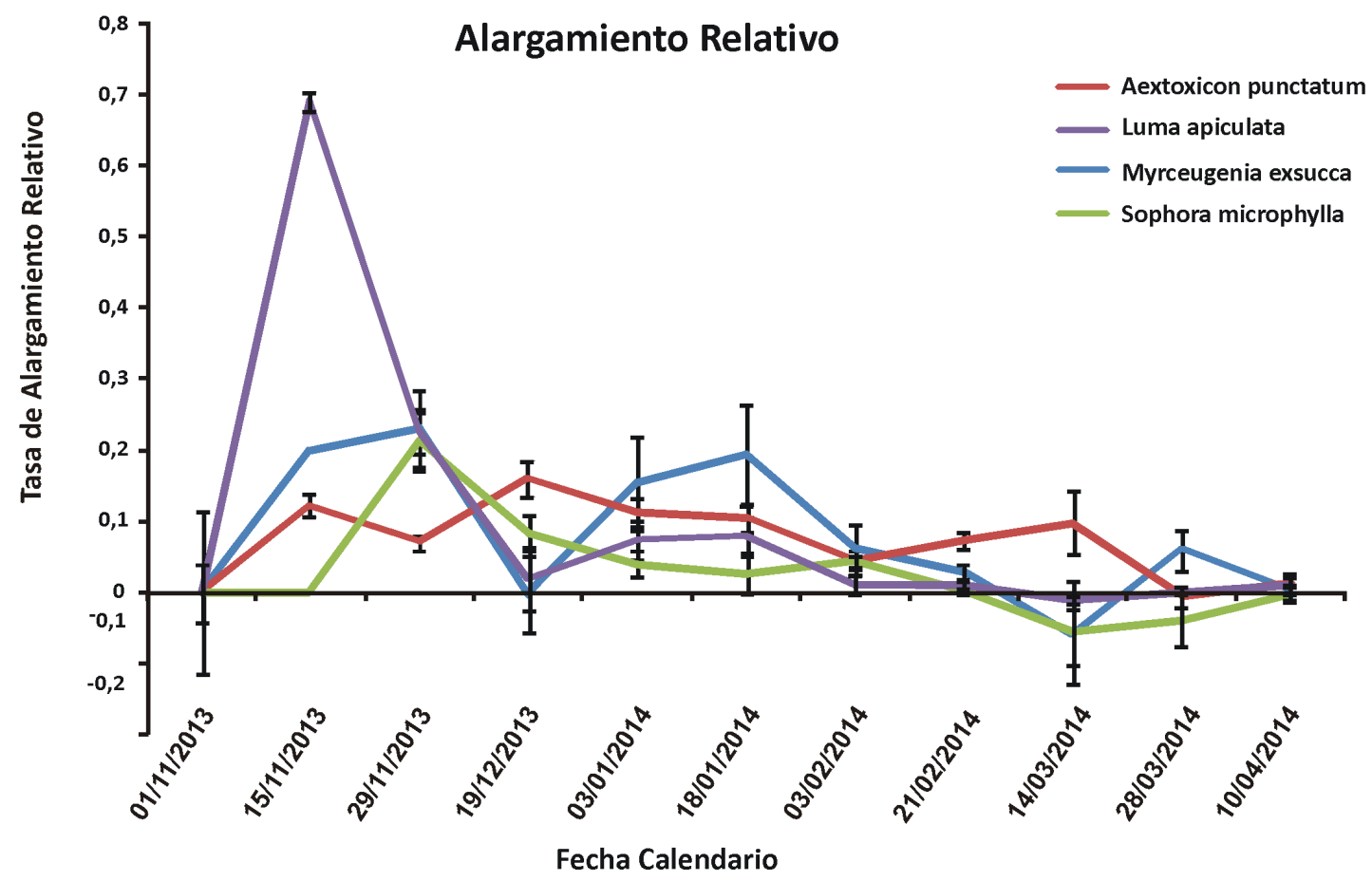

Fig. 4. Promedio $( \pm E E)$ de la tasa de alargamiento relativo a lo largo del tiempo para brotes anuales de Aextoxicon punctatum, Luma apiculata, Myrceugenia exsucca y Sophora microphylla.

2013 seguido de una caída muy pronunciada, luego de la cual el crecimiento se mantuvo bajo hasta fines de enero de 2014 (Fig. 4).

En $M$. exsucca, el inicio de alargamiento se produjo entre la primera quincena de noviembre de 2013 y la primera de enero de 2014 (Fig. 3E). La finalización ocurrió entre la primera quincena de noviembre de 2013 y la primera de abril de 2014 (Fig. 3E). La duración de alargamiento varió entre 1 y 9 quincenas (Fig. 3F). La mayoría de los individuos de $M$. exsucca presentaron un pico en la TAR en noviembre, seguido de una caída notable en diciembre. Se registró un segundo pico de alargamiento, menos generalizado y de menor magnitud que el primero, entre fines de diciembre y enero. Posteriormente, la TAR se fue reduciendo gradualmente, registrándose en muchos casos valores negativos debidos a muertes de ápice con desecación de entrenudos distales y, en consecuencia, disminuciones en la longitud de los brotes (Fig. 4).

En S. microphylla, el inicio de alargamiento se produjo entre la segunda quincena de noviembre y la primera quincena de diciembre de 2013 (Fig. $3 \mathrm{G})$. Las fechas de finalización abarcaron entre la primera quincena de diciembre de 2013 y la primera de abril de 2014 (Fig. 3G). La duración del alargamiento fue como mínimo de 2 y como máximo de 9 quincenas (Fig. 3H). La mayoría de los individuos de $S$. microphylla presentaron un pico en la TAR en la segunda mitad de noviembre, seguido de un descenso notable en semanas subsecuentes; se registró un segundo pico de baja magnitud en la primera quincena de febrero de 2014. En marzo de 2014 se registraron valores negativos de la TAR debido a la alta frecuencia de muertes de ápices y de entrenudos distales (Fig. 4).

Al comparar las TAR de forma inter-específica se destacan, por un lado, el elevado valor promedio registrado para L. apiculata al inicio del período de crecimiento, y por otro lado la escasa variación de esta variable para A. punctatum.

Los promedios de temperatura diurna y nocturna y de humedad relativa del ambiente diurna y nocturna fueron, respectivamente, de $10,9{ }^{\circ} \mathrm{C}$ y $7,2{ }^{\circ} \mathrm{C}$ y de $72,5 \%$ y $85,7 \%$ para el año completo de mediciones, 
y de $16,1{ }^{\circ} \mathrm{C}$ y $10,4{ }^{\circ} \mathrm{C}$ y $59,7 \%$ y $79,1 \%$ para el período de crecimiento de las plantas. Además, las temperaturas mínimas diurnas y nocturnas fueron, respectivamente, de $4,3{ }^{\circ} \mathrm{C}$ y $3,6^{\circ} \mathrm{C}$, y las máximas diurnas y nocturnas de $24,3{ }^{\circ} \mathrm{C}$ y $16,8{ }^{\circ} \mathrm{C}$ para el período de crecimiento de las plantas.

\section{Discusión}

En este estudio se comprueba que las especies valdivianas $A$. punctatum, L. apiculata, M. exsucca y $S$. microphylla presentan crecimiento primario en el período primavera-verano (mayormente entre noviembre y marzo), aunque con diferencias intrae inter-específicas en la dinámica de crecimiento. Estas diferencias pueden considerarse notables si se toman en cuenta la edad similar de las plantas consideradas y la aparente homogeneidad del ambiente en el cual se desarrollaron las plantas estudiadas.

La duración del período de alargamiento resultó muy variable entre las cuatro especies estudiadas. La especie que presentó menor variabilidad intraespecífica en la duración del crecimiento fue A. punctatum. La variación en la duración del crecimiento fue máxima para $L$. apiculata y $M$. exsucca e intermedia para $S$. microphylla.

Para A. punctatum y $S$. microphylla, las fechas de inicio de alargamiento fueron poco variables, lo cual concuerda con los resultados de estudios previos sobre numerosas especies de otras regiones (Kozlowski \& Ward, 1961) y sobre especies patagónicas de Nothofagus (Puntieri et al., 1998; Stecconi et al., 2000). Desde hace varias décadas se conoce que el inicio del crecimiento primario rítmico de las plantas está determinado principalmente por factores endógenos y por aquellos factores ambientales que varían en forma regular siguiendo ciclos anuales, como el fotoperíodo y la temperatura media (Kramer \& Kozlowski, 1960; Mitchell, 1965; Kozlowski, 1971; Gratani, 1996; Schreiber et al., 2013). Se destaca la gran variabilidad intra-específica en el inicio del alargamiento (dos meses o más) para las dos especies de Myrtaceae estudiadas aquí, $L$. apiculata y $M$. exsucca. Estas variaciones intraespecíficas son superiores a las registradas para otras especies leñosas de Patagonia (Puntieri et al., 1998; Stecconi et al., 2000; Puntieri et al., 2002), más aún si se toma en cuenta la aparente homogeneidad ambiental en el vivero. Se ha detectado que el inicio de brotación de Metrosideros polymorpha Gaudich., una especie de Myrtaceae de zonas tropicales, puede ocurrir en cualquier momento del año (Gerrish, 1989). Las variaciones intraespecíficas en el inicio del crecimiento primario podrían justificarse aludiendo a la existencia de varios factores o de combinaciones de factores. Esos factores o combinaciones de factores serían de naturaleza tal que podrían afectar a algunas y no a todas las plantas de una misma población, quizás debido a diferencias genéticas entre éstas (e.g. Walter \& Soos, 1963). El estudio de la dinámica del crecimiento de ejemplares genéticamente caracterizados de varias especies de Myrtaceae bajo condiciones controladas contribuiría a determinar el origen de la variabilidad intra-específica en el inicio del crecimiento primario.

La finalización del crecimiento estacional también responde a factores endógenos y exógenos, pero entre estos últimos se incluyen factores de ocurrencia menos regular o imprevisible, como por ejemplo heladas tempranas (Puntieri et al., 1998; Stecconi et al., 2002). La finalización del alargamiento de un brote puede estar influenciada principalmente por: (1) el vigor de la planta, (2) el número de órganos preformados del brote, (3) la capacidad del brote de desarrollar órganos neoformados, y (4) el ambiente en el cual se encuentra (Kozlowski, 1964; Puntieri et al., 2000; Puntieri et al., 2002; Guédon et al., 2006; Schreiber et al., 2013). Si bien faltan estudios sobre esta temática, se conoce para otras especies que existen vínculos estrechos entre estos factores. Por ejemplo, el vigor de un eje en plantas de Nothofagus pumilio (Poepp. \& Endl.) Krasser (lenga), N. dombeyi (Mirb.) Oersted (coihue) y N. antarctica incide en la preformación que ese eje es capaz de desarrollar en sus yemas y en la probabilidad de que ese eje desarrolle órganos neoformados (Souza et al., 2000; Puntieri et al., 2002). Podría argumentarse, en principio, que las especies para las cuales se registraron mayores variaciones en las fechas de finalización del crecimiento ( $L$. apiculata, $M$. exsucca y $S$. microphylla) responderían a más de uno de estos factores. Pero hasta el momento se desconoce en qué medida varían la preformación y la neoformación de órganos en estas especies. Por otro lado, la relativamente poco variable finalización 
del crecimiento registrada para $A$. punctatum es acorde con resultados obtenidos en un estudio previo realizado sobre otros ejemplares en el mismo vivero (Gerea, 2008). En base a la información sobre otras especies de regiones templadas y, en particular, de los bosques patagónicos (Guédon et al., 2006), podría suponerse que los brotes de $A$. punctatum que se desarrollaron en las plantas medidas habrían derivado exclusivamente de órganos que se encontraban preformados en las yemas de invierno.

La tasa de alargamiento relativo de los brotes mostró amplias variaciones intra- e inter-específicas. Para las cuatro especies estudiadas, esa tasa alcanzó su valor máximo en las primeras semanas del alargamiento (entre noviembre y diciembre), como se ha observado en estudios anteriores sobre brotes de especies de Nothofagus de la Patagonia (Puntieri et al., 1998; Stecconi et al., 2000). Aextoxicon punctatum mantuvo una tasa baja pero relativamente homogénea hasta fines del verano, mientras que $M$. exsucca, L. apiculata y S. microphylla exhibieron fluctuaciones mayores. Cabe destacar que ambas especies de la familia Myrtaceae mostraron una caída de la tasa de alargamiento a mediados de diciembre y un repunte en enero. Estas variaciones se debieron, en parte, a las ya mencionadas variaciones intraespecíficas en el inicio del crecimiento, pero también podrían relacionarse con el patrón de alargamiento de los brotes. Se ha encontrado en varias especies arbóreas de regiones templadas que la ocurrencia del pico de alargamiento máximo próximo al momento de la brotación de primavera y de un segundo pico menos importante en verano se vinculan, respectivamente, con la expansión de los órganos preformados y la de órganos neoformados (Guédon et al., 2006). Para otras especies, la ocurrencia de varios picos de alargamiento se vincula con la formación de unidades de alargamiento sucesivas en la misma estación de crecimiento (brotes policíclicos; Barthélémy \& Caraglio, 2007). Nuestros resultados sugieren que las variaciones registradas aquí para la tasa de alargamiento relativo de L. apiculata y M. exsucca resultarían, dependiendo de la planta considerada, de la ocurrencia de uno o dos eventos de morfogénesis. En todas estas plantas, el inicio del crecimiento estacional corresponde al alargamiento de órganos diferenciados previamente a esa estación (primer evento de morfogénesis). Un segundo evento de morfogénesis se habría producido sólo en aquellas plantas en las cuales se formó una rama relevo luego de la muerte del ápice en crecimiento (Fig. 2C). La muerte del ápice de un brote en crecimiento seguida de la formación de un relevo a partir de la brotación de una yema axilar del mismo brote es un fenómeno de ocurrencia regular en muchas especies (Barthélémy \& Caraglio, 2007). Las observaciones realizadas en este estudio sugieren que en los casos de L. apiculata y M. exsucca la muerte apical con formación de relevo se vincularía a factores exógenos, algo también sugerido para otras especies arbóreas patagónicas (e.g. Puntieri et al., 1998, 2007).

\section{Crecimiento primario y distribución}

Los amplios márgenes de finalización del crecimiento primario, y los períodos de crecimiento extensos registrados aquí para $L$. apiculata, $M$. exsucca y $S$. microphylla, así como el amplio margen de inicio del crecimiento en las dos primeras de estas especies, sugieren que la dinámica de crecimiento de estas plantas respondería a un conjunto de factores ambientales. Si bien nuestros resultados no prueban fehacientemente que el crecimiento de estas especies sea potencialmente continuo, aportan evidencia en este sentido. Por el contrario, el crecimiento de $A$. punctatum estuvo concentrado en un período de tiempo más limitado, lo que sugiere la existencia de un control endógeno acorde con el crecimiento calificado de rítmico. De las cuatro especies estudiadas, A. punctatum es la que presenta los primordios más expuestos durante la fase de inicio del alargamiento, a diferencia de lo que ocurre en las otras tres especies, en las cuales sólo los primordios más externos quedan expuestos (Fig. 1). Se podría proponer que en A. punctatum se compensa la mayor exposición de los primordios con un crecimiento más delimitado en el tiempo, lo que posibilita que sus hojas nuevas alcancen pleno desarrollo en el período del año más favorable.

Las familias Myrtaceae y Fabaceae se encuentran ampliamente diversificadas en regiones tropicales y subtropicales de Sudamérica (Mabberley, 2009). Los géneros Luma y Myrceugenia pertenecen a la tribu Myrteae, y sus especies más meridionales habrían derivado de representantes de un grupo actualmente diversificado a menor latitud en Chile (Murillo et al., 2012; Vasconcelos et al., 2015). En el caso del género Sophora, posee distribución pantropical; $S$. microphylla habría evolucionado a partir de especies 


\section{M. Sosa y J. G. Puntieri - Crecimiento anual en especies arbóreas de Patagonia}

procedentes de zonas más cálidas de Sudamérica (Peña et al., 2000). En cuanto a A. punctatum, pertenece a una familia monoespecífica con relaciones evolutivas poco claras (APG III, 2009), y habita ambientes húmedos y templado-cálidos, como lo sugiere su distribución actual en Chile (NúñezÁvila \& Armesto, 2006). De modo que la posesión de yemas desnudas en las especies patagónicas incluidas en estos géneros es acorde con su origen evolutivo en regiones con bajos niveles de restricción al crecimiento por bajos niveles de temperatura y humedad. La selva valdiviana es el bioma patagónico que más se aproxima a esas condiciones. Las posibilidades de expansión de estas especies en otras regiones de la Patagonia estarían limitadas por la ocurrencia de condiciones ambientales más severas. Más aún, su sobrevivencia en la selva valdiviana podría peligrar si se produjeran cambios ambientales que significaran bajos niveles de humedad durante primavera y verano, como prevén proyecciones recientes sobre cambio climático para esta región (Barros et al., 2015; Cabré et al., 2016; Ruscica et al., 2016).

\section{Conclusión}

Las especies A. punctatum, L. apiculata, M. exsucca y $S$. microphylla comparten con numerosas especies de regiones tropicales y subtropicales la posesión de yemas apicales desnudas. Como en otras especies de regiones templadas, el alargamiento de sus brotes se produce principalmente en el período primavera-verano, algo que concuerda con el crecimiento rítmico. No obstante, las amplias variaciones intra-específicas registradas para el inicio y la finalización del crecimiento de $L$. apiculata y $M$. exsucca y, en menor medida, de S. microphylla, sugieren la intervención de varios factores en la delimitación del período de crecimiento de estas especies, un atributo acorde con el crecimiento continuo.

\section{Agradecimientos}

A Rubén Pablos (Asociación Civil Sembrar), por brindarnos el lugar y las plantas utilizadas en el estudio. A la Administración del Parque Nacional Lago Puelo, y en especial a Félix Vidoz, Nadia
Zermatten y Marcos Ocampo, quienes posibilitaron las visitas a poblaciones naturales de las especies estudiadas. A Eduardo Gómez de Olivera, Marina Stecconi, Cristian Torres, Amaru Magnin, Javier Grosfeld, Anahí Currulef y Brisa Guenuleo por sus colaboraciones en diferentes instancias de este estudio. A CONICET por la beca (C.S.) durante la realización de este trabajo.

\section{Bibliografía}

AIZEN, M. A. \& C. EZCURRA. 1998. High incidence of plant-animal mutualisms in the woody flora of the temperate forest of southern South America: biogeographical origin and present ecological significance. Ecol. Austral 8: 217-236.

APG III. 2009. An update of the Angiosperm Phylogeny Group classification for the orders and families of flowering plants. Bot. J. Linn. Soc. 161: 105-121.

BARROS, V. R., J. A. BONINSEGNA, I. A. CAMILLONI, M. CHIDIAK, G. O. MAGRÍN \& M. RUSTICUCCI. 2015. Climate change in Argentina: trends, projections, impacts and adaptation. WIREs Clim. Change 6: 151169.

BARTHÉLÉMY, D. \& Y. CARAGLIO. 2007. Plant architecture: a dynamic, multilevel and comprehensive approach to plant form, structure and ontogeny. Ann. Bot. 99: 375-407.

BARTHÉLÉMY, D., J. PUNTIERI, C. BRION, E. RAFFAELE, J. MARINO \& P. MARTINEZ. 1999. Características morfológicas y arquitecturales de las especies de Nothofagus Blume (Fagaceae) del norte de la Patagonia Argentina. Bol. Soc. Argent. Bot. 34: 29-38.

CABRÉ, M. F., S. SOLMAN \& M. NÚÑEZ. 2016. Regional climate change scenarios over southern South America for future climate (2080-2099) using the MM5 Model. Mean, interannual variability and uncertainties. Atmosfera 1. Disponible en: http://www.redalyc.org/ jatsRepo/565/56544662004/56544662004_visor_jats. pdf. [Acceso: 13 septiembre 2016].

CABRERA, A. L. \& A. WILLINK. 1980. Biogeografía de América Latina. Serie Biológica. Monografía $n^{\circ} 13$. O.E.A., Washington D.C.

CONTI, H. A. 1998. Características climáticas de la Patagonia. En: CORREA, M. N. (ed.), Flora Patagónica Vol. VIII (I), pp. 31-47. INTA, Buenos Aires.

DONOSO, C. 1993. Bosques templados de Chile y Argentina. 4ta edición. Editorial Universitaria, Santiago de Chile.

DONOSO, C. 2006. Las especies arbóreas de los bosques templados de Chile y Argentina. Autoecología. Marisa Cuneo Ediciones, Valdivia.

EZCURRA, C. \& C. BRION. 2005. Plantas del Nahuel Huapi. Catálogo de la Flora Vascular del Parque Nacional 
Nahuel Huapi, Argentina. Red Latinoamericana de Botánica, Universidad Nacional del Comahue, San Carlos de Bariloche.

GERRISH, G. 1989. Comparing crown growth and phenology of juvenile, early mature, and late mature Metrosideros polymorpha trees. Pac. Sci. 43: 211222.

GEREA, M. 2008. Dinámica de alargamiento de los ejes en especies de la Flora Valdiviana. Tesina de Licenciatura en Ciencias Biológicas, Universidad Nacional del Comahue, San Carlos de Bariloche.

GRATANI, L. 1996. Leaf and shoot growth dynamics of Quercus ilex L. Acta Oecol. 17: 17-27.

GROSFELD, J. \& D. BARTHÉLÉMY. 2004. Primary growth and morphological markers of interannual growth limits in Cupressaceae from Patagonia. Bot. J. Linn. Soc. 146: 285-293.

GUÉDON, Y., J. PUNTIERI, S. SABATIER \& D. BARTHÉLÉMY. 2006. Relative extents of preformation and neoformation in tree shoots: analysis by a deconvolution method. Ann. Bot. 98: 835-844.

HALLÉ, F., R. A. A. OLDEMAN \& P. B. TOMLINSON. 1978. Tropical trees and forests. An architectural analysis. Springer-Verlag, Berlín.

KOZLOWSKI, T. T. 1964. Shoot growth in woody plants. Bot. Rev. 30: 335-392.

KOZLOWSKI, T. T. 1971. Growth and development of trees, vol I: Seed germination, ontogeny and shoot growth. Acad. Press, New York.

KOZLOWSKI, T. T. \& R. C. WARD. 1961. Shoot elongation characteristics of forest trees. Forest Sci. 7: 357-368.

KRAMER, P. J. \& T. T. KOZLOWSKI. 1960. Physiology of trees. McGraw-Hill, New York.

LATORRE, C., P. MORENO, G. VARGAS, A. MALDONADO, R. VILLA-MARTÍNEZ, J. J. ARMESTO, C. VILLAGRÁN, M. PINO \& M. GROSJEAN. 2007. Late Quaternary environments and palaeoclimate. En: LATORRE, C. (ed.), The Geology of Chile, pp. 309-328. The Geological Society of London, Londres.

MABBERLEY, D. J. 2009. Mabberley's Plant-Book, A portable dictionary of plants, their classification and uses. Cambridge University Press, New York.

MAGNIN, A., J. GROSFELD, D. BARTHÉLÉMY \& J. PUNTIERI. 2012. Bud and shoot structure may relate to the distribution area of South American Proteaceae tree species. Flora 207: 599-606.

MITCHELL, A. F. 1965. The growth in early life of the leading shoot of some conifers. Forestry 38: 121136.

MURILLO-A, J., E. RUIZ-P., L. R. LANDRUM, T. F. STUESSY \& M. H. J. BARFUSS. 2012. Phylogenetic relationships in Myrceugenia
(Myrtaceae) based on plastid and nuclear DNA sequences. Mol. Phylogenet. Evol. 62: 764-776.

MUÑOZ SCHICK, M. 1980. Flora del Parque Nacional Puyehue. Editorial Universitaria, Santiago de Chile.

NUÑEZ-AVILA, M. C. \& J. J. ARMESTO. 2006. Relict islands of the temperate rainforest tree Aextoxicon punctatum (Aextoxicaceae) in semi-arid Chile: genetic diversity and biogeographic history. Australian J. Bot. 54: 733-743.

PEÑA, R. C., L. ITURRIAGA, G. MONTENEGRO \& B. K. CASSELS. 2000. Phylogenetic and biogeographic aspects of Sophora Sect. Edwardsia (Papilionaceae). Pac. Sci. 54: 159-167.

PUNTIERI, J., D. BARTHÉLÉMY, P. MARTINEZ, E. RAFFAELE \& C. BRION. 1998. Annual-shoot growth and branching patterns in Nothofagus dombeyi (Fagaceae). Can. J. Bot. 76: 673-685.

PUNTIERI, J., M. S. SOUZA, D. BARTHÉLÉMY, C. BRION, C. NUÑEZ \& C. MAZZINI. 2000. Preformation, neoformation and shoot structure in Nothofagus dombeyi (Nothofagaceae). Can. J. Bot. 78: 1044-1054.

PUNTIERI, J., D. BARTHÉLÉMY, C. MAZZINI \& C. BRION. 2002. Periods of organogenesis in shoot of Nothofagus dombeyi (Mirb.) Oersted (Nothofagaceae). Ann. Bot. 89: 115-124.

PUNTIERI, J., J. GROSFELD, M. STECCONI, M. M. AZPILICUETA, L. GALLO, C. BRION \& D. BARTHÉLÉMY. 2007. Shoot development and dieback in progenies of Nothofagus obliqua. Ann. For. Sci. 64: 839-844.

RUSCICA, R.,C. C. G. MENÉNDEZ \& A. A. SÖRENSSON. 2016. Land surface- atmosphere interaction in future South American climate using a multi-model ensemble. Atmos. Sci. Let. 17: 141147.

SCHREIBER, G. S., A. HAMANN, U. G. HACKE \& B. R. THOMAS. 2013. Sixteen years of winter stress: an assessment of cold hardiness, growth performance and survival of hybrid poplar clones at a boreal planting site. Plant Cell Environ. 36: 419-428.

SOUZA, M. S., J. PUNTIERI, D. BARTHÉLÉMY \& C. BRION. 2000. Bud content and its relation to shoot and structure in Nothofagus pumilio (Poepp. et Endl.) Krasser (Nothofagaceae). Ann. Bot. 85: 547-555.

STECCONI, M. 2006. Variabilidad Arquitectural de especies nativas de Nothofagus de la Patagonia (N. antarctica, N. pumilio, N. dombeyi). Tesis de Doctorado en Ciencias Biológicas, Universidad Nacional del Comahue, San Carlos de Bariloche.

STECCONI, M., J. PUNTIERI \& D. BARTÉLÉMY. 2000. Annual shoot-growth in Nothofagus antarctica (G. Forster) Oersted (Nothofagaceae) from northern Patagonia. Trees 14: 289-296. 


\section{M. Sosa y J. G. Puntieri - Crecimiento anual en especies arbóreas de Patagonia}

STECCONI, M., P. MARCHELLI, J. PUNTIERI \& L. GALLO. 2002. Hybridisation between Nothofagus antarctica (deciduous) and $N$. dombeyi (evergreen) (Nothofagaceae) in natural communities. South. Connection Bull. 21: 8-9.

VASCONCELOS, T. N. C., G. PRENNER, M. O. BÜNGER, P. S. DE-CARVALHO, A. WINGLER \& E. J. LUCAS. 2015. Systematic and evolutionary implications of stamen position in Myrteae (Myrtaceae). Bot. J. Linn. Soc. 179: 388-402.
WALTER, J. \& L. SOOS. 1963. Shoot growth patterns of some British Columbia conifers. Forest Sci. 9: 73-85.

Recibido el 20 de abril de 2016, aceptado el 15 de septiembre de 2016. 
